\title{
CARAFE++: Unified Content-Aware ReAssembly of FEatures
}

\author{
Jiaqi Wang, Kai Chen, Rui Xu, Ziwei Liu, Chen Change Loy, and Dahua Lin
}

\begin{abstract}
Feature reassembly, i.e. feature downsampling and upsampling, is a key operation in a number of modern convolutional network architectures, e.g., residual networks and feature pyramids. Its design is critical for dense prediction tasks such as object detection and semantic/instance segmentation. In this work, we propose unified Content-Aware ReAssembly of FEatures (CARAFE++), a universal, lightweight and highly effective operator to fulfill this goal. CARAFE++ has several appealing properties: (1) Unlike conventional methods such as pooling and interpolation that only exploit sub-pixel neighborhood, CARAFE++ aggregates contextual information within a large receptive field. (2) Instead of using a fixed kernel for all samples (e.g. convolution and deconvolution), CARAFE++ generates adaptive kernels on-the-fly to enable instance-specific content-aware handling. (3) CARAFE++ introduces little computational overhead and can be readily integrated into modern network architectures. We conduct comprehensive evaluations on standard benchmarks in object detection, instance/semantic segmentation and image inpainting. CARAFE++ shows consistent and substantial gains across all the tasks $\left(2.5 \% A P_{b o x}, 2.1 \% A P_{\text {mask }}, 1.94 \% \mathrm{mloU}, 1.35 \mathrm{~dB}\right.$ respectively) with negligible computational overhead. It shows great potential to serve as a strong building block for modern deep networks.
\end{abstract}

Index Terms-Feature Reassembly, Object Detection, Instance Segmentation, Semantic Segmentation, Image Inpainting.

\section{INTRODUCTION}

$\mathrm{F}$ EATURE reassembly, i.e., downsampling and upsampling, is one of the most fundamental operations in deep neural networks. On the one hand, in dense prediction tasks (e.g., super resolution [8], [22], inpainting [15], [34] and semantic segmentation |5|, |47|), the input image is downsampled in the encoders to enlarge receptive field, gather semantic information, and reduce computational cost. For the decoders, the high-level/low-resolution feature map is upsampled to match the high-resolution supervision. On the other hand, feature reassembly is also involved in fusing a high-level/low-resolution feature map with a lowlevel/high-resolution feature map, which is widely adopted in many state-of-the-art architectures, e.g., Feature Pyramid Network [23], U-Net [36] and Stacked Hourglass [32]. Therefore, designing an effective feature reassembly operator becomes a critical issue.

Feature reassembly is composed of downsampling and upsampling on feature maps. Pooling and interpolation are the most widely adopted operator families for downsampling and upsampling, respectively. The max pooling and average pooling are two representative pooling methods, which reassemble features inside a local region with a hand-crafted kernel. The nearest neighbor and bilinear interpolations are the most commonly used upsampling

- Jiaqi Wang is with The Chinese University of Hong Kong, Hong Kong. E-mail:wj017@ie.cuhk.edu.hk.

- Chen Kai is with The Chinese University of Hong Kong, Hong Kong. E-mail: ck015@ie.cuhk.edu.hk.

- Rui Xu is with The Chinese University of Hong Kong, Hong Kong. Email:xr018@ie.cuhk.edu.hk.

- Ziwei Liu is with Nanyang Technological University, Singapore. E-mail: ziwei.liu@ntu.edu.sg.

- Chen Change Loy is with Nanyang Technological University, Singapore. E-mail: ccloy@ntu.edu.sg.

- Dahua Lin is with The Chinese University of Hong Kong, Hong Kong. E-mail:dhlin@ie.cuhk.edu.hk.
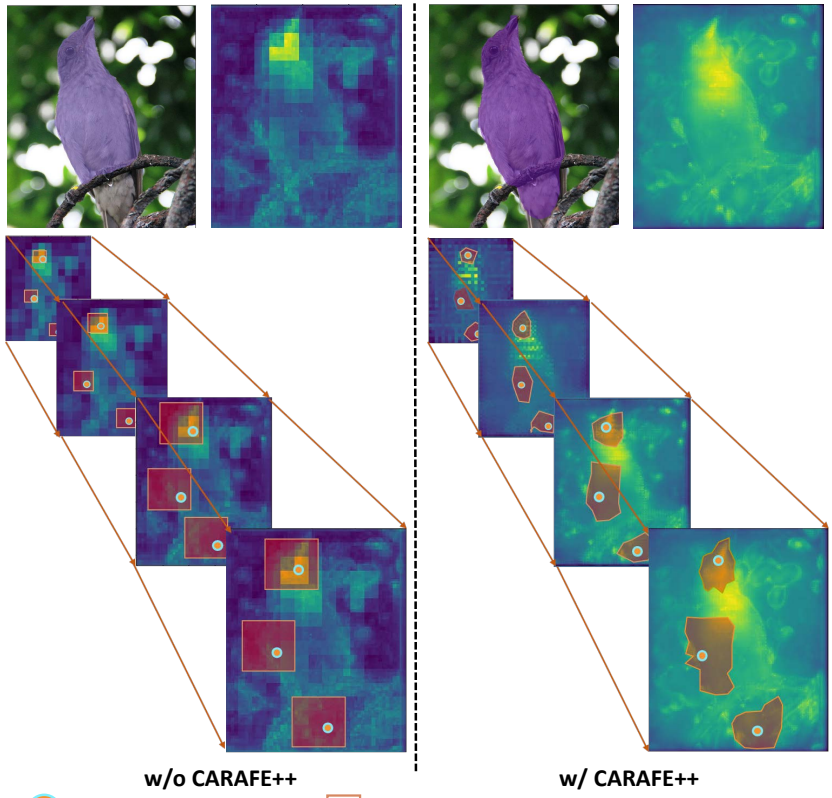

w/ CARAFE++

Example Location

Fig. 1: Illustration of CARAFE++ working mechanism. Left: Multi-level FPN features from Mask R-CNN baseline (left to dotted line) and Right: Multi-level FPN features from Mask R-CNN with CARAFE++(right to dotted line). For sampled locations, this figure shows the accumulated reassembled regions in the top-down pathway of FPN. Information inside such a region is reassembled into the corresponding reassembly center.

operators, which adopt spatial distance between pixels to guide the upsampling process. However, both pooling and interpolation are rule-based operators, which fail to capture the rich semantic information required by dense prediction tasks.

An alternative method for adaptive feature reassembly is convolution and deconvolution [33]. A convolution layer 
adjusts 'stride' to control the spatial distance to apply convolution kernel. A deconvolution layer works as an inverse operator of a convolution layer, which learns a set of instance-agnostic upsampling kernels. However, there exist two major drawbacks. First, a convolution/deconvolution operator applies the same kernel across the entire image, regardless of the underlying content. This restricts its capability in responding to local variations. Second, it comes with heavy computational workload when a large kernel size is used. This makes it difficult to cover a larger region that goes beyond a small neighborhood, thus limiting its expressive power and performance.

In this work, we move beyond these limitations, and seek a feature reassembly operator that is capable of 1) aggregating information within large receptive field, 2) adapting to instance-specific contents on-the-fly, and 3) maintaining computation efficiency. To this end, we propose a lightweight yet highly effective operator, called unified Content-Aware ReAssembly of Features (CARAFE++). Specifically, CARAFE++ reassembles the features inside a predefined region via a weighted combination, where the weights are generated in a content-aware manner. A reassembly kernel is generated for each target location. And the feature reassembly is performed on the corresponding location of the input feature map.

Note that these spatially adaptive weights are not learned as network parameters. Instead, they are predicted on-the-fly, using a lightweight fully-convolutional module with softmax activation. Figure 1 reveals the working mechanism of CARAFE++ in an upsampling case. We visualize the feature maps in the top-down pathway of feature pyramid network (FPN) |23| and compare CARAFE++with the nearest neighbor interpolation baseline. After upsampled by CARAFE++, a feature map can represent the shape of an object more accurately, so that the model can predict better instance segmentation results. Our CARAFE++ not only rescales the feature map spatially, but also learns to enhance its discrimination.

To demonstrate the universal effectiveness of CARAFE ++ , we conduct comprehensive evaluations across a wide range of dense prediction tasks, i.e., object detection, instance segmentation, semantic segmentation, image inpainting, with mainstream architectures. With negligible extra cost, CARAFE++ boosts the performance of Faster R-CNN [35] by $2.5 \% A P_{b o x}$ in object detection and Mask R-CNN [12] by 2.1\% $A P_{\text {mask }}$ in instance segmentation on MS COCO |25| test-dev. CARAFE++ further improves UperNet [43] by 1.94\% mIoU on ADE20k [51], [52] val in semantic segmentation, and improves Global\&Local [15] by $1.35 \mathrm{~dB}$ of PSNR on Places [50] val in image inpainting. The substantial gains on all the tasks demonstrate that CARAFE++ is an effective and efficient feature reassembly operator that has great potential to serve as a strong building block for future research.

In a previous conference version in ICCV 2019, we proposed CARAFE [40], which is an effective and efficient upsampling operator. CARAFE++ shares a similar design while it is more universal. CARAFE++ can be readily integrated to networks for both upsampling and downsampling. We perform an extensive evaluation of CARAFE++ when it is applied in a wide range of net- work architectures. Experimental results show that adopting CARAFE++ in both upsampling and downsampling can consistently and substantially outperforms CARAFE 11 on object detection, instance segmentation, semantic segmentation and image inpainting.

\section{Related Work}

Downsampling \& Upsampling Operators. Downsampling \& upsampling operators are basic building blocks of convolutional neural network architectures. Contemporary convolution networks usually downsample the input features in the first few layers. Feature upsampling operators are essential for convolutional networks to make high-quality dense predictions.

Among various downsampling operators, max pooling and average pooling are the most widely used choices. They reassemble features in a rule-based manner. For upsampling, nearest neighbor and bilinear interpolations are representative methods. These interpolations leverage distances to measure the correlations between pixels, and handcrafted upsampling kernels are used in them. Several methods are proposed to downsample and upsample a feature map using learnable operators. For example, convolution and deconvolution (transposed convolution) $|33|$ are the most representative ones among those learnable upsamplers. Pixel Shuffle [38] proposes an upsampling operator that reshapes depth of the channel space into width and height of the spatial space. Guided Upsampling (GUM) |30| performs interpolation by sampling pixels with learnable offsets. In the downsampling pipeline, detail-preserving pooling (DPP) [37] is proposed to preserve more detail information by focusing on local spatial changes of pixels in a sliding window. Local Importance-based Pooling (LIP) [9] is a recently proposed downsampling operator, which performs a weighted average pooling. However, these methods either exploit contextual information in a small neighborhood, or require expensive computation to perform adaptive downsampling and upsampling. Within the realms of super-resolution and denoising, some other works [14], [18], [31 also explore the use of learnable kernels spatially in lowlevel vision. In this study, we demonstrate the effectiveness and working mechanism of unified content-aware feature reassembly for downsampling and upsampling in several visual perception tasks, and provide a lightweight solution. Dense Prediction Tasks. Object detection [24], [28], [35], [41], [42] is the task of localizing objects with boundingboxes, instance segmentation [12] further requires the prediction of instance-wise masks. Many studies [19], [23], [27], [49] exploit multi-scale feature pyramids to deal with objects at different scales. By adding extra mask prediction branches, Mask R-CNN [12] and its variants [1] yield promising pixel-level results. Semantic segmentation [21], [29] requires pixel-wise semantic prediction for given images. PSPNet [47] introduces spatial pooling at multiple grid scales. UperNet [43] designs a more generalized framework based on PSPNet. These detection and segmentation methods usually adopt backbones, e.g., ResNet [13], which are pretrained on image classification dataset. The input

1. Adopting CARAFE is equivalent to adopting CARAFE++ for upsampling. 


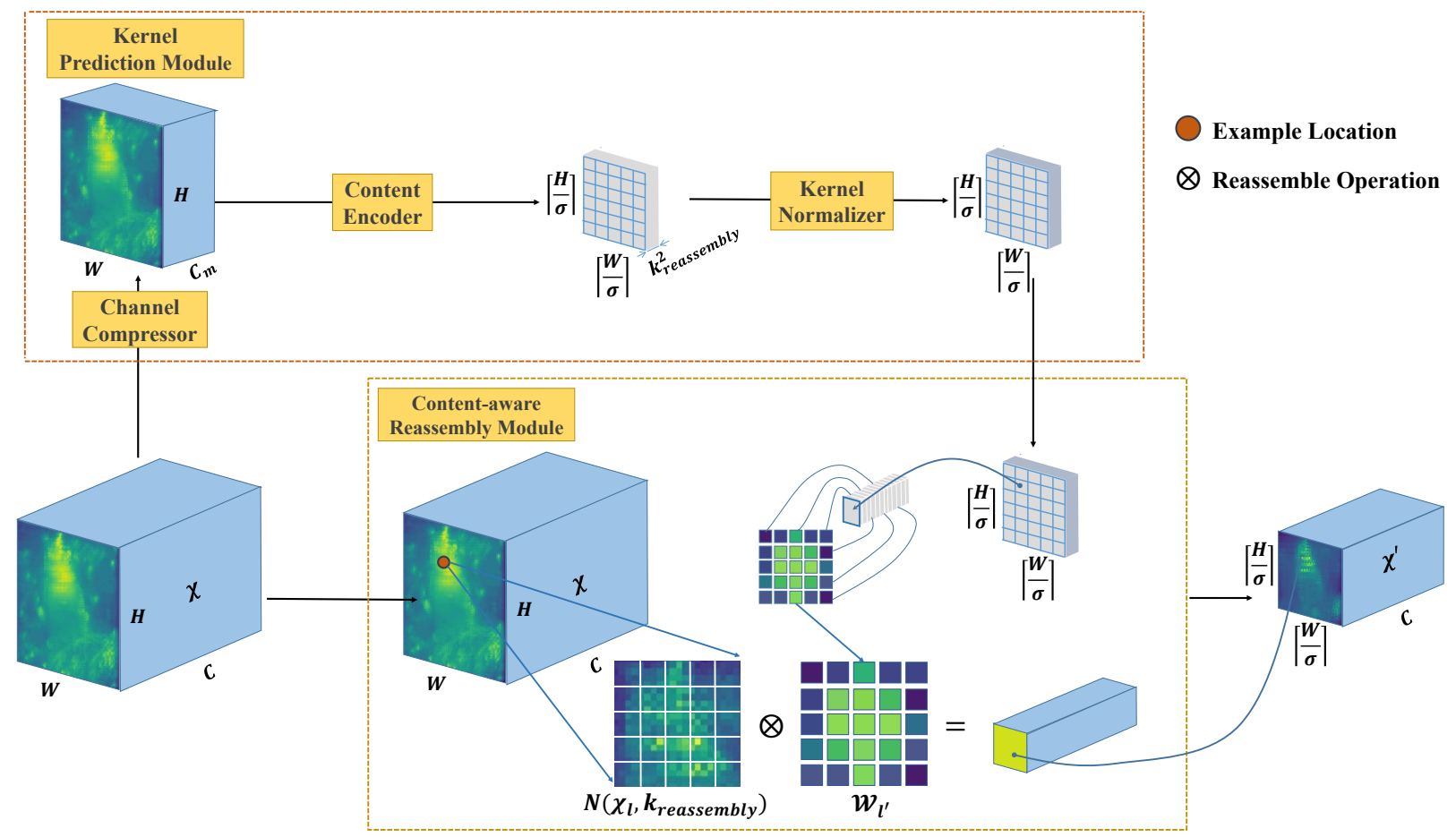

Fig. 2: The overall framework of CARAFE++ for downsampling. CARAFE++ is composed of two key components, i.e., kernel prediction module and content-aware reassembly module. A feature map with size $C \times H \times W$ is downsampled by a factor of $\sigma(=2)$ in this figure.

features are downsampled for several times in such image classification networks. Image or video inpainting [39], [44], [46] is a classical problem that aims at completing missing regions of images. U-net [36], which adopts multiple downsampling and upsampling operators, is popular among recent works [15], [39]. Liu et al. [26] introduce partial convolution layer to alleviate the influence of missing regions on the convolution layers. By replacing the downsampling and upsampling operators in the aforementioned networks, our CARAFE++ demonstrates consistent effectiveness across a wide range of dense prediction tasks.

\section{Content-Aware ReAssembly of FEatures}

Feature reassembly, i.e., downsampling, upsampling, is a key operator in many modern convolutional network architectures developed for tasks including object detection, instance segmentation, and scene parsing. In this work, we propose Content-Aware ReAssembly of FEatures (CARAFE++) to reassemble a feature map. On each location, CARAFE++ leverages the underlying content information to predict reassembly kernels and reassemble the features inside a predefined nearby region. Thanks to this unique capability, CARAFE++ achieves better performance than the mainstream downsampling and upsampling operators, e.g., pooling or interpolation.

\subsection{Formulation}

CARAFE++ works as a reassembly operator with contentaware kernels. It consists of two steps. The first step is to predict a reassembly kernel for each target location according to its content, and the second step is to reassemble the features with the predicted kernels.
Given a feature map $\mathcal{X}$ of size $C \times H \times W$ and a resizing ratio $\sigma$ (supposing $\sigma$ is an integer), CARAFE++ will produce a new feature map $\mathcal{X}^{\prime}$ of size $C \times\lceil H / \sigma\rceil \times\lceil W / \sigma\rceil$ in the downsampling process and $C \times \sigma H \times \sigma W$ in the upsampling process. For any target location $l^{\prime}=\left(i^{\prime}, j^{\prime}\right)$ of the output $\mathcal{X}^{\prime}$, there is a corresponding source location $l=(i, j)$ at the input $\mathcal{X}$, where $i=\sigma i^{\prime}, j=\sigma j^{\prime}$ for downsampling, and $i=\left\lfloor i^{\prime} / \sigma\right\rfloor, j=\left\lfloor j^{\prime} / \sigma\right\rfloor$ for upsampling. Here we denote $N\left(\mathcal{X}_{l}, k\right)$ as the $k \times k$ sub-region of $\mathcal{X}$ centered at the location l, i.e., the neighbor of $\mathcal{X}_{l}$.

In the first step, the kernel prediction module $\psi$ predicts a location-wise kernel $\mathcal{W}_{l^{\prime}}$ for each location $l^{\prime}$, based on the neighbor of $\mathcal{X}_{l}$, as shown in Eqn. (1). The reassembly step is formulated as Eqn. (2), where $\phi$ is the content-aware reassembly module that reassembles the neighbor of $\mathcal{X}_{l}$ with the kernel $\mathcal{W}_{l^{\prime}}$ :

$$
\begin{gathered}
\mathcal{W}_{l^{\prime}}=\psi\left(N\left(\mathcal{X}_{l}, k_{\text {encoder }}\right)\right) . \\
\mathcal{X}_{l^{\prime}}^{\prime}=\phi\left(N\left(\mathcal{X}_{l}, k_{\text {reassembly }}\right), \mathcal{W}_{l^{\prime}}\right) .
\end{gathered}
$$

We specify the details of $\psi$ and $\phi$ in the following parts.

\subsection{Kernel Prediction Module}

The kernel prediction module is responsible for generating the reassembly kernels in a content-aware manner. Each target location corresponds to a source location and requires a $k_{\text {reassembly }} \times k_{\text {reassembly }}$ reassembly kernel, where $k_{\text {reassembly }}$ is the reassembly kernel size. Therefore, this module will output the reassembly kernels of size $C_{\text {reassembly }} \times\lceil H / \sigma\rceil \times\lceil W / \sigma\rceil$ for downsampling, and $C_{\text {reassembly }} \times \sigma H \times \sigma W$ for upsampling, where $C_{\text {reassembly }}=k_{\text {reassembly }}^{2}$.

The kernel prediction module is composed of three submodules, i.e., channel compressor, content encoder and kernel 


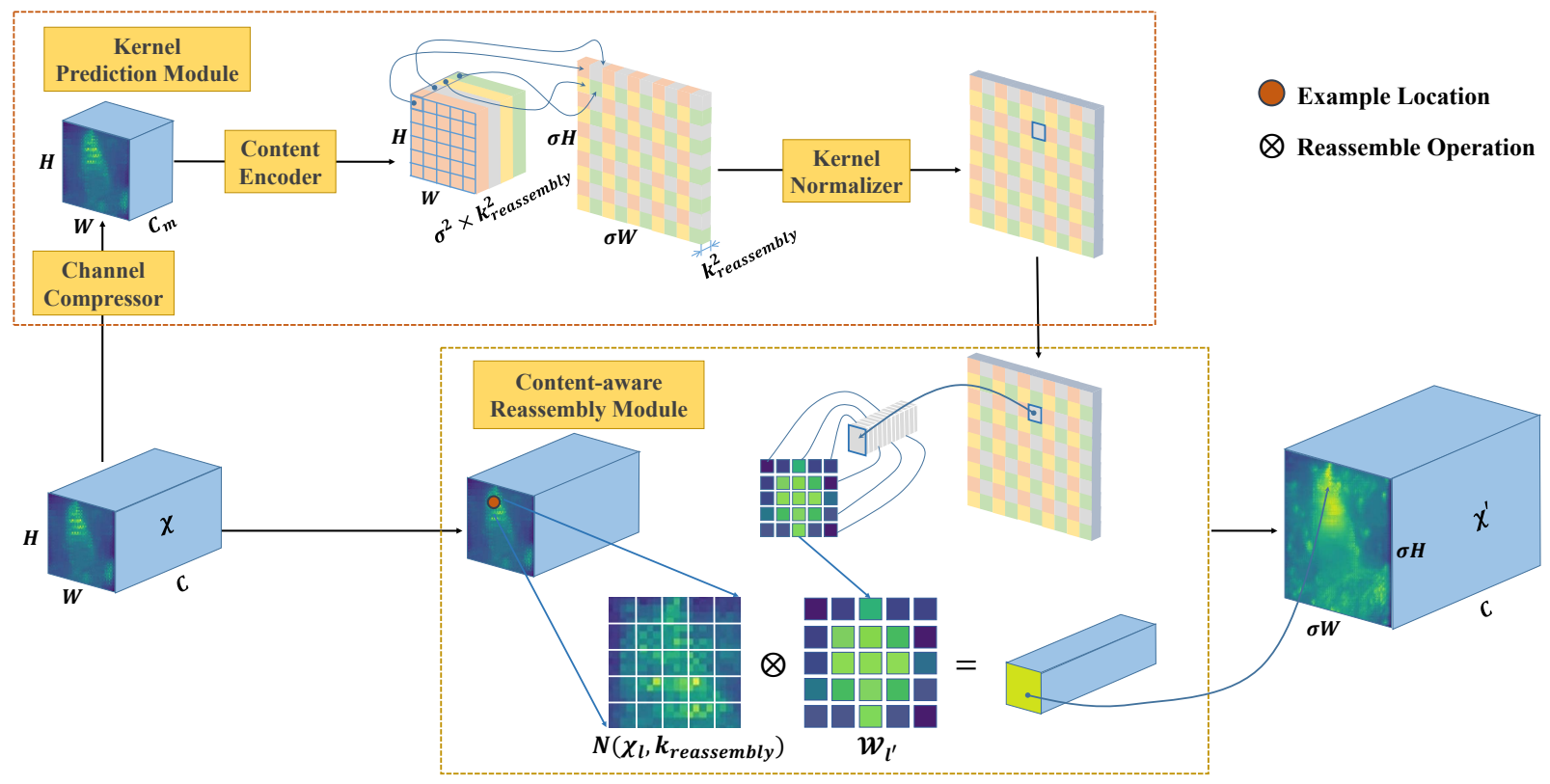

Fig. 3: The overall framework of CARAFE++ for upsampling. A feature map with size $C \times H \times W$ is upsampled by a factor of $\sigma(=2)$ in this figure.

normalizer, as shown in Figure 2 and Figure 3 . The channel compressor reduces the channel of the input feature map. The content encoder then takes the compressed feature map as input and encodes the content to generate reassembly kernels. Lastly, the kernel normalizer applies a softmax function to each reassembly kernel. The three submodules are explained in detail as follows.

Channel Compressor. We adopt a $1 \times 1$ convolution layer to compress the input feature channel from $C$ to $C_{m}$. Specifically, we adopt $C_{m}=16$ for downsampling and $C_{m}=64$ for upsampling in experiments. Reducing the channel of input feature map leads to less computational cost in the following steps, making CARAFE++ much more efficient. It is also possible to use larger kernel sizes for the content encoder under the same budget. Experimental results show that reducing the feature channel in an acceptable range will not harm the performance.

Content Encoder. We use a convolution layer of kernel size $k_{\text {encoder }}$ to generate reassembly kernels based on the content of input features. The input channel of this convolution layer is $C_{m}$. Certain settings are different for downsampling and upsampling.

For downsampling, the stride of this convolution layer is $\sigma$. Thus, the predicted reassembly kernel has a size of $C_{\text {reassembly }} \times\lceil H / \sigma\rceil \times\lceil W / \sigma\rceil$. For upsampling, the output channels of this convolution layer is $\sigma^{2} C_{\text {reassembly. It is then }}$ reorganized from depth of the channel space into width and height of the spatial space. Finally, the predicted reassembly kernel has a size of $C_{\text {reassembly }} \times \sigma H \times \sigma W$.

Intuitively, increasing $k_{\text {encoder }}$ enlarges the receptive field of the encoder, and this allows one to exploit contextual information within a larger region, which is important for predicting the reassembly kernels. However, the computational complexity grows quadratically with the kernel size, while the benefits from a larger kernel size may not match equally. An empirical formula, $k_{\text {encoder }}=k_{\text {reassembly }}-2$, is a good trade-off between performance and efficiency through our study in Section 5.3

Kernel Normalizer. Before being applied to the input feature map, each reassembly kernel with size of $k_{\text {reassembly }} \times$ $k_{\text {reassembly }}$ is normalized with a softmax function spatially. The normalization step forces the sum of kernel values to 1 . The normalized values result a soft selection across a local region. Due to the kernel normalizer, CARAFE++ does not perform any rescaling and change to the mean values of the feature map, but reassembles features spatiallly.

\subsection{Content-aware Reassembly Module}

With each reassembly kernel $\mathcal{W}_{l^{\prime}}$, the content-aware reassembly module reassembles the features within a local region via the function $\phi$. We adopt a simple form of $\phi$, which is just a weighted sum operator. For a target location $l^{\prime}$ and the corresponding square region $N\left(\mathcal{X}_{l}, k_{\text {reassembly }}\right)$ centered at $l=(i, j)$, the reassembly is shown in Eqn. (3), where $r=\left\lfloor k_{\text {reassembly }} / 2\right\rfloor$ :

$$
\mathcal{X}_{l^{\prime}}^{\prime}=\sum_{n=-r}^{r} \sum_{m=-r}^{r} \mathcal{W}_{l^{\prime}(n, m)} \cdot \mathcal{X}_{(i+n, j+m)} .
$$

With the reassembly kernel, each pixel in the region of $N\left(\mathcal{X}_{l}, k_{\text {reassembly }}\right)$ contributes to the target pixel $l^{\prime}$ differently, based on the content of features. The semantics of the reassembled feature map can be stronger than the original one, since the information from relevant points in a local region can be more attended.

\subsection{Relation to Previous Operators}

Here we discuss the relations between CARAFE++ and dynamic filter [17], spatial attention [3], local importancebased pooling |9|, spatial transformer |16] and deformable convolution $|6|$, which share similar design philosophy but with different focuses.

Dynamic Filter. Dynamic filter generates instance-specific convolutional filters conditioned on the input of the network, and then applies the predicted filter on the input. Both 
dynamic filter and CARAFE++ are content-aware operators, but a fundamental difference between them lies at their kernel generation process. Specifically, dynamic filter works as a two-step convolution, where the additional dynamic filter prediction step requires heavy computation. On the contrary, CARAFE++ is simply a reassembly of features in local regions, without learning the feature transformation across channels. Supposing the channels of input feature map is $C$ and kernel size of the filter is $K$, then the predicted kernel weights for each location is $C \times C \times K \times K$ in dynamic filter. For CARAFE++, the kernel weights is only $K \times K$. Thus, it is more efficient in memory and speed.

Spatial Attention. Spatial attention predicts an attention map with the same spatial size as the input feature, and then rescales the feature map on each location. Our CARAFE++ reassembles the features in a local region by weighted summation. In summary, spatial attention is a rescaling operator with point-wise guidance while CARAFE ++ is a reassembly operator with region-wise local guidance. Spatial attention can be seen as a special case of CARAFE++ where the reassembly kernel size is 1 , regardless of the kernel normalizer.

Local Importance-Based Pooling (LIP). Local ImportanceBased Pooling (LIP) is a recently proposed pooling method, which combines the self-attention and average pooling. In LIP, an attention map with the same shape (i.e., spatial size and channels) as the input feature map is predicted by a series of convolution layers. The predicted attention map is normalized by a sigmoid function, and then directly multiplied by the original feature map. A standard average pooling downsamples the feature map after self-attention. The self-attention in LIP is a combination of spatial attention and channel attention. Similar to spatial attention, it fails to adaptively reassemble information from a large receptive field. To predict the attention map with the same shape as the input, LIP requires a series of convolution layers with deep output channels and brings heavy computational cost. Moreover, LIP is not available for upsampling.

Spatial Transformer Networks (STN). STN predicts a global parametric transformation conditioned on the input feature map and warps the feature via the transformation. However, this global parametric transformation assumption is too strong to represent complex spatial variance; and STN is known to be instable to train. Here, CARAFE++ uses the location-specific reassembly to handle the spatial relations, which enables more flexible local geometry modeling.

Deformable Convolutional Networks (DCN). DCN also adopts the idea of learning geometric transformation and combines it with the regular convolution layers. It predicts kernel offsets other than using grid convolution kernels. Similar to dynamic filter, it is also a heavy parametric operator with much more computational cost than CARAFE++.

\section{Applications OF CARAFE++}

CARAFE++ can be seamlessly integrated into existing frameworks where downsampling \& upsampling operators are needed. Here we present some applications in mainstream dense prediction tasks. With negligible additional computational cost, CARAFE++ benefits mainstream methods in both high-level and low-level tasks, such as object de-

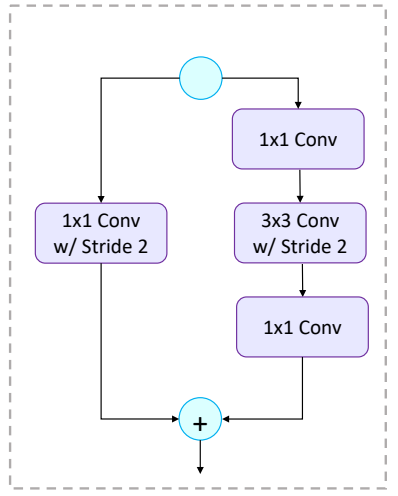

Bottleneck w/ Strided Conv

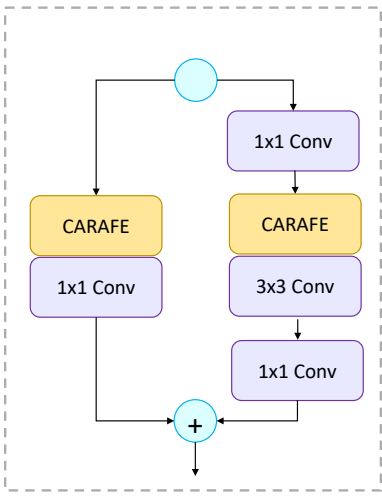

Bottleneck w/ CARAFE
Fig. 4: Bottleneck architecture with CARAFE++ in ResNet. CARAFE++ can be readily integrated into Bottleneck with strided convolution. CARAFE++ downsamples the input feature map by $2 x$. And convolution layers with the same kernel size but no stride are applied afterwards.

tection, instance segmentation, semantic segmentation and image inpainting.

\subsection{Object Detection and Instance Segmentation}

ResNet Backbone. Backbone is the cornerstone of object detection. The backbone with strided convolution layers is adopted to downsample input images and produce pyramid features. The backbone applied in object detection and instance segmentation is generally pretrained on image classification dataset, e.g., ImageNet.

ResNet backbone [13] is one of the most widely used backbones in object detection. ResNet has five blocks, named as Res-1, Res-2, Res-3, Res-4 and Res-5, respectively. Res- 1 contains one $3 \times 3$ convolution layer and one max pooling layer, it is also called as the stem layer. Res2, Res-3, Res-4 and Res-5 are stacked building blocks. A feature map is downsampled in Res-3, Res-4 and Res-5 at their first building block with strided convolution layers. CARAFE ++ can be readily integrated into ResNet. As shown in Figure 4. CARAFE++ is applied to the building block (named as Bottleneck) with strided convolution layers. In the original design of bottleneck, convolution layers with stride downsamples the feature map by $2 x$ directly. In the bottleneck $w$ / CARAFE++, CARAFE++ downsamples the feature map before convolution layers. Furthermore, CARAFE++ replaces the max pooling which downsamples the feature map by $2 x$ in Res- 1 as well. In summary, there are seven CARAFE++ are applied in a ResNet backbone, i.e., one in Res-1, two in each of Res-3, Res-4 and Res-5.

Feature Pyramid Network (FPN). FPN is an important and effective architecture in the field of object detection and instance segmentation. It significantly improves the performance of popular frameworks like Faster R-CNN and Mask R-CNN. FPN constructs feature pyramids of strong semantics with the top-down pathway and lateral connections. In the top-down pathway, a low-resolution feature map is firstly upsampled by $2 x$ with the nearest neighbor interpolation and then fused with a high-resolution one, as shown in Figure 5 We propose to substitute the nearest neighbor interpolation in all the feature levels with CARAFE++. This modification is smooth and no extra change is required. 


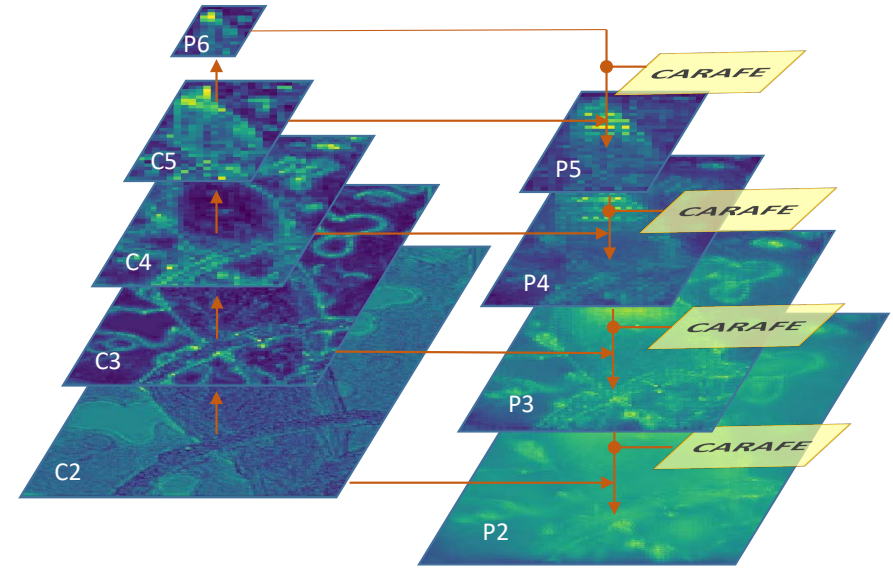

Fig. 5: FPN architecture with CARAFE++. CARAFE++ upsamples a feature map by a factor of 2 in the top-down pathway. It is integrated into FPN by seamlessly substituting the nearest neighbor interpolation.

Mask Head. In addition, Mask R-CNN adopts a deconvolution layer at the end of mask head. It is used to upsample the predicted digits from $14 \times 14$ to $28 \times 28$, to obtain finer mask predictions. We can also use CARAFE ++ to replace the deconvolution layer, resulting in even less computational cost.

\subsection{Semantic Segmentation}

Semantic segmentation requires the model to output perpixel level predictions on the whole image, so that highresolution feature maps are usually preferred. In a common pipeline of semantic segmentation model, the pretrained backbone, e.g., ResNet, with downsampling layers are first applied to downsample the feature maps. And then upsampling is widely adopted to enlarge feature maps from the backbone and fuse the semantic information of different levels in this task. UperNet is a strong baseline for semantic segmentation. It uses downsampling in ResNet backbone and upsampling in the following three components, i.e., PPM, FPN, FUSE. We adopt CARAFE++ instead of their original downsamplers and upsamplers.

ResNet Backbone. CARAFE++ is adopted as in Section 4.1 i.e., Res- 1 and first building block of Res-3, Res-4, Res-5.

Pyramid Pooling Module (PPM). PPM is the key component in PSPNet that hierarchically down-samples an input feature map into multiple scales $\{1 \times 1,2 \times 2,3 \times 3,6 \times 6\}$, and then upsamples them back to the original sizes with bilinear interpolation. The features are finally fused with the original feature by concatenation. Since the upsampling ratio is very large, we adopt a two-step strategy with CARAFE++ as a trade-off between performance and efficiency. Firstly we upsamples the $\{1 \times 1,2 \times 2,3 \times 3,6 \times 6\}$ features to half the size of the original feature map with bilinear interpolation, and then use CARAFE++ to further upsample them by $2 x$.

Feature Pyramid Network (FPN). Similar to detection models, UperNet also adopts FPN to enrich the feature semantics. It only has four different feature levels $\{\mathrm{P} 2, \mathrm{P} 3, \mathrm{P} 4$, P5 $\}$ with strides $\{4,8,16,32\}$. We replace the upsampling operators in the same way as Section 4.1

Multi-level Feature Fusion (FUSE). UperNet introduces a multi-level feature fusion module after the FPN. It upsam- ples P3, P4, P5 to the same size as P2 by bilinear interpolation and then fuses these features from different levels by concatenation. The process is equivalent to a sequential upsampling-concatenation that first upsamples P5 to P4 and concatenates them, and then upsamples the concatenated feature map to P3 and so on. We replace the sequential bilinear upsampling here with CARAFE++.

\subsection{Image Inpainting}

The U-net architecture is popular among recently proposed image inpainting methods, such as Global\&Local [15] and Partial Conv [26]. We simply replace the strided convolution layers with CARAFE++ followed by convolution layer without stride, and also substitute upsampling layers with CARAFE++ . As for Partial Conv, we can conveniently keep the mask propagation in CARAFE++ by updating the mask with our content-aware reassembly kernels.

\section{EXPERIMENTS}

\subsection{Experimental Settings}

Datasets \& Evaluation Metrics. We evaluate CARAFE++ on several important dense prediction benchmarks. We use the train split for training and evaluate the performance on the val split for all these datasets if it is not further specified. The inference speed is reported on a single TiTan XP GPU. Image Classification. To evaluate CARAFE++ on the backbone of dense prediction tasks, we pretrain backbones on ImageNet-1k [7] train split and evaluate the Top-1 and Top5 accuracy on val split with the single-crop testing.

Object Detection and Instance Segmentation. We perform experiments on the challenging MS COCO 2017 [25] dataset. Results are evaluated with the standard COCO metric, i.e. AP of IoUs from 0.5 to 0.95 .

Semantic Segmentation. We adopt the ADE20k [51], [52] benchmark to evaluate our method in the semantic segmentation task. Results are measured with mean IoU (mIoU) and Pixel Accuracy (P.A.), which respectively indicates the average IoU between predictions and ground truth masks and per-pixel classification accuracy.

Image Inpainting. Places [50| dataset is adopted for image inpainting. We use L1 error (lower is better) and PSNR (higher is better) as evaluation metrics.

Implementation Details. If not otherwise specified, CARAFE++ adopts a fixed set of hyper-parameters in experiments, where $C_{m}$ is 16 and 64 for the channel compressor in downsampling and upsampling, respectively. And $k_{\text {encoder }}=3, k_{\text {reassembly }}=5$ for the content encoder.

Image Classification. We evaluate CARAFE++ on ResNet-50 and ResNet-101 backbones. As described in Section 4.1. CARAFE++ is applied in Bottleneck with strided convolution layers in Res-3, Res-4, Res-5, and replaces the max pooling in Res-1. Furthermore, a BatchNorm (BN) and a Relu layer are adopted after the channel compressor, which is a $1 \times 1$ Conv that compresses input channels. The experimental settings mostly follow [11]. We use 16 GPUs with batch size of 1024, i.e., 64 images per GPU. We train backbones for 90 epochs in total on ImageNet-1k classification dataset.

Object Detection and Instance Segmentation. We evaluate CARAFE++ on Faster R-CNN [35] and Mask R-CNN [12] 
TABLE 1: Detection and Instance Segmentation results on MS COCO 2017 test-dev with 2x training schedule.

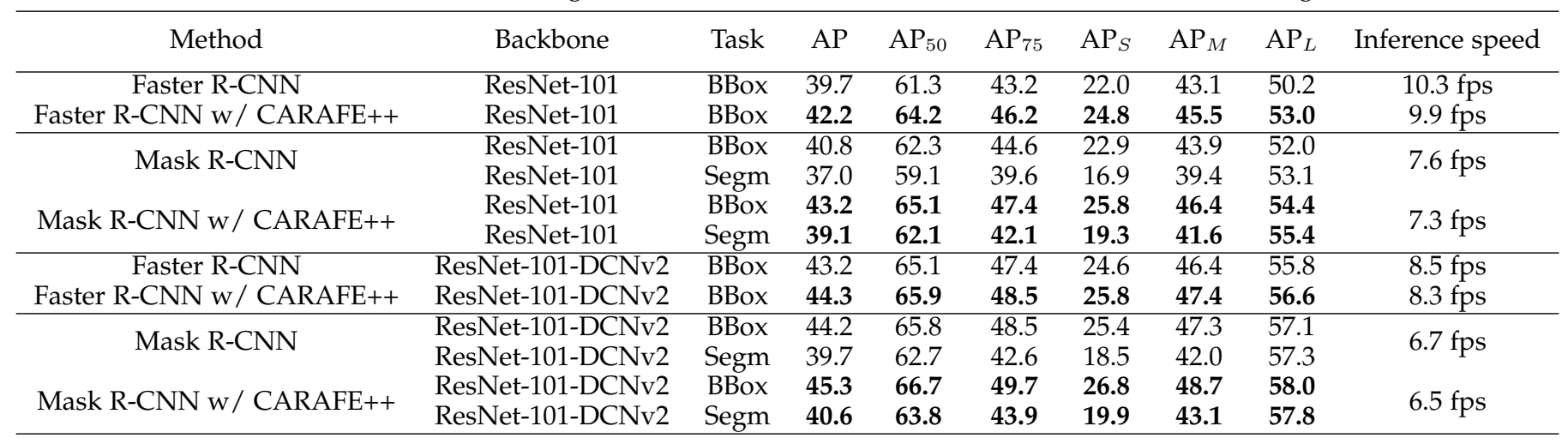
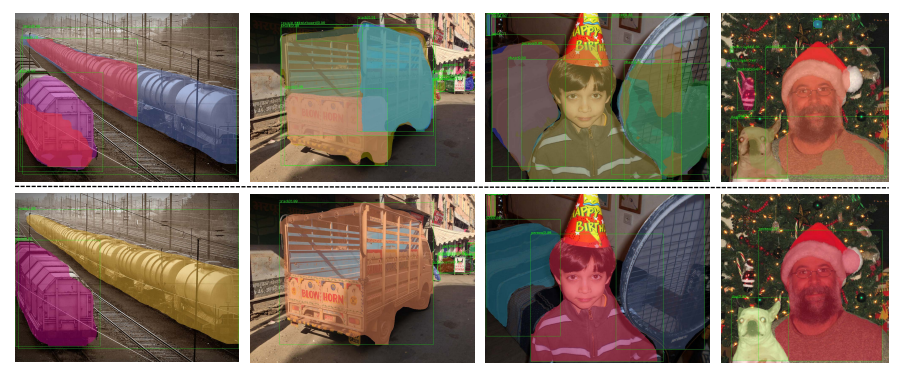

Fig. 6: Comparison of instance segmentation results between baseline (top row) and CARAFE++ (bottom row) on COCO 2017 val.

TABLE 2: Image classification results on ImageNet-1k val.

\begin{tabular}{ccc}
\hline Model & Top-1 & Top-5 \\
\hline ResNet-50 & 76.43 & 93.21 \\
ResNet-50 w/CARAFE++ & $\mathbf{7 7 . 4 6}$ & 93.63 \\
\hline ResNet-101 & 78.10 & 93.92 \\
ResNet-101 w/ CARAFE++ & $\mathbf{7 8 . 7 0}$ & 94.31 \\
\hline
\end{tabular}

with the ResNet-50 [13] w/ FPN [23] backbone for ablation study and report the test-dev results with ResNet-101 backbones. In both training and inference, we resize an input image such that its shorter edge has 800 pixels or longer edge has 1333 pixels without changing its aspect ratio. We adopt synchronized SGD with an initial learning rate of 0.02 , a momentum of 0.9 and a weight decay of 0.0001 . We use a batchsize of 16 over 8 GPUs (2 images per GPU). Following the $1 x$ and $2 x$ training schedule as Detectron [10] and MMDetection [2].

Semantic Segmentation. We use the official implementation of UperNet [43] with the ResNet backbone as the baseline. During the training, an input image is resized such that the size of its shorter edge is randomly selected from $\{300,375$, $450,525,600\}$. In inference, we apply the single scale testing for a fair comparison and the shorter edge of an image is set to 450 pixels. The maximum length of the longer edge of an image is set to 1200 in both training and inference. We adopt synchronized SGD with an initial learning rate of 0.02 , a momentum of 0.9 and a weight decay of 0.0001 . We use a batchsize of 16 over 8 GPUs ( 2 images per GPU), and synchronized batch normalization is adopted as a common practice in semantic segmentation. Following [4], the 'poly' learning rate policy is adopted. And we train the model for 20 epochs.

Image Inpainting. We adopt Global\&Local [15] as the baseline
TABLE 3: Detection results with Faster R-CNN. Various downsampling methods are used in ResNet-50 backbone. 'Conv', 'MPool', 'APool', 'DPP', 'LIP' indicates convolution layer with stride, Max Pooling, Average Pooling, Detail Preserved Pooling [37], and Local Importance-based Pooling [9], respectively. The $1 \mathrm{x}$ training schedule is applied in experiments.

\begin{tabular}{cccccccc}
\hline Method & $\mathrm{AP}_{\text {box }}$ & $\mathrm{AP}_{50}$ & $\mathrm{AP}_{75}$ & $\mathrm{AP}_{S}$ & $\mathrm{AP}_{M}$ & $\mathrm{AP}_{L}$ & \multirow{2}{*}{$\mathrm{FPS}$} \\
\hline Conv & 36.5 & 58.4 & 39.3 & 21.3 & 40.3 & 47.2 & $12.5 \mathrm{fps}$ \\
Max Pool & 37.8 & 59.6 & 40.9 & 22.3 & 42.1 & 48.4 & $12.5 \mathrm{fps}$ \\
Avg Pool & 37.9 & 59.8 & 40.9 & 22.1 & 42.1 & 47.9 & $12.3 \mathrm{fps}$ \\
DPP [37] & 37.7 & 59.7 & 40.6 & 22.3 & 41.9 & 48.1 & $10.9 \mathrm{fps}$ \\
LIP |9] & 38.0 & 60.3 & 41.2 & 23.1 & 41.9 & 48.2 & $10.8 \mathrm{fps}$ \\
CARAFE++ & $\mathbf{3 8 . 8}$ & 60.8 & 42.2 & 23.3 & 43.1 & 49.2 & $12.1 \mathrm{fps}$ \\
\hline
\end{tabular}

TABLE 4: Detection results with Faster R-CNN. Various upsampling methods are used in FPN. N.C., B.C., P.S. and S.A. indicate Nearest + Conv, Bilinear + Conv, Pixel Shuffle and Spatial Attention, respectively.

\begin{tabular}{cccccccc}
\hline Method & $\mathrm{AP}_{\text {box }}$ & $\mathrm{AP}_{50}$ & $\mathrm{AP}_{75}$ & $\mathrm{AP}_{S}$ & $\mathrm{AP}_{M}$ & $\mathrm{AP}_{L}$ & $\mathrm{FPS}$ \\
\hline Nearest & 36.5 & 58.4 & 39.3 & 21.3 & 40.3 & 47.2 & $12.5 \mathrm{fps}$ \\
Bilinear & 36.7 & 58.7 & 39.7 & 21.0 & 40.5 & 47.5 & $12.5 \mathrm{fps}$ \\
N.C. & 36.6 & 58.6 & 39.5 & 21.4 & 40.3 & 46.4 & $11.8 \mathrm{fps}$ \\
B.C. & 36.6 & 58.7 & 39.4 & 21.6 & 40.6 & 46.8 & $11.7 \mathrm{fps}$ \\
Deconv [33] & 36.4 & 58.2 & 39.2 & 21.3 & 39.9 & 46.5 & $11.5 \mathrm{fps}$ \\
P.S. [38] & 36.5 & 58.8 & 39.1 & 20.9 & 40.4 & 46.7 & $11.7 \mathrm{fps}$ \\
GUM [30] & 36.9 & 58.9 & 39.7 & 21.5 & 40.6 & 48.1 & $11.8 \mathrm{fps}$ \\
S.A. [3] & 36.9 & 58.8 & 39.8 & 21.7 & 40.8 & 47.0 & $12.3 \mathrm{fps}$ \\
CARAFE++ & $\mathbf{3 7 . 8}$ & 60.1 & 40.8 & 23.2 & 41.2 & 48.2 & $12.2 \mathrm{fps}$ \\
\hline
\end{tabular}

method to evaluate CARAFE++. We employ the generator and discriminator networks from Global\&Local [15]. Our generator takes a $256 \times 256$ image $\mathrm{x}$ with masked region $M$ as input and produces a $256 \times 256$ prediction of the missing region $\hat{\mathbf{y}}$ as output. Then we combine the predicted image with the input by $\mathbf{y}=(1-M) \odot \mathbf{x}+M \odot \hat{\mathbf{y}}$. Finally, the combined output $\mathrm{y}$ is fed into the discriminator. We apply a simple modification to the baseline model to achieve better generation quality. Compared to the original model that employs two discriminators, we employ only one PatchGANstyle discriminator [20] on the inpainted region. For a fair comparison, we use the free-form masks introduced by [45] as the binary mask $M$. We further compare CARAFE++with Partial Conv [26], we substitute the convolution layers with the official Partial Conv module in our generator. During training, Adam solver with learning rate 0.0001 is adopted where $\beta_{1}=0.5$ and $\beta_{2}=0.9$. Training batch size is 32 . The input and output are linearly scaled within range $[-1,1]$. 
TABLE 5: Object detection results of Faster R-CNN with CARAFE++ in FPN and backbone.

\begin{tabular}{ccccccccc}
\hline \multirow{2}{*}{ FPN } & Backbone & $\mathrm{AP}$ & $\mathrm{AP}_{50}$ & $\mathrm{AP}_{75}$ & $\mathrm{AP}_{S}$ & $\mathrm{AP}_{M}$ & $\mathrm{AP}_{L}$ & \multirow{2}{*}{$\mathrm{FPS}$} \\
\hline$\checkmark$ & & 37.8 & 60.1 & 40.8 & 23.2 & 41.2 & 48.2 & $12.2 \mathrm{fps}$ \\
& $\checkmark$ & 38.8 & 60.8 & 42.2 & 23.3 & 43.1 & 49.2 & $12.1 \mathrm{fps}$ \\
$\checkmark$ & $\checkmark$ & $\mathbf{3 9 . 6}$ & 62.0 & 43.2 & 24.7 & 43.7 & 50.9 & $11.7 \mathrm{fps}$ \\
\hline
\end{tabular}

TABLE 6: Instance Segmentation results with Mask R-CNN. Various upsampling methods are used in mask head.

\begin{tabular}{ccccccc}
\hline Method & $\mathrm{AP}$ & $\mathrm{AP}_{50}$ & $\mathrm{AP}_{75}$ & $\mathrm{AP}_{S}$ & $\mathrm{AP}_{M}$ & $\mathrm{AP}_{L}$ \\
\hline Nearest & 32.7 & 55.0 & 34.8 & 17.7 & 35.9 & 44.4 \\
Bilinear & 34.2 & 55.9 & 36.4 & 18.5 & 37.5 & 46.2 \\
Deconv & 34.2 & 55.5 & 36.3 & 17.6 & 37.8 & 46.7 \\
Pixel Shuffle & 34.4 & 56.0 & 36.6 & 18.5 & 37.6 & 47.5 \\
GUM & 34.3 & 55.7 & 36.5 & 17.6 & 37.6 & 46.9 \\
S.A. & 34.1 & 55.6 & 36.5 & 17.6 & 37.4 & 46.6 \\
CARAFE++ & $\mathbf{3 4 . 7}$ & 56.2 & 37.1 & 18.2 & 37.9 & 47.5 \\
\hline
\end{tabular}

\subsection{Benchmarking Results}

Object Detection \& Instance Segmentation. We first evaluate CARAFE++ in Faster R-CNN and Mask R-CNN to show its effectiveness in object detection and instance segmentation. We first replace the downsampling layers in ResNet-101 backbone with CARAFE++ and pretrain the modified backbone in ImageNet-1k classification dataset as in Section 4.1. We then substitute the nearest neighbor interpolation in FPN with CARAFE++ for both Faster R-CNN and Mask R-CNN, and the deconvolution layer in the mask head for Mask R-CNN. As shown in Table 1, with ResNet101 backbone and $2 x$ training schedule, CARAFE++ improves Faster R-CNN by $2.5 \%$ (i.e., from $39.7 \%$ to $42.2 \%$ ) on $A P_{b o x}$, and Mask R-CNN by $2.1 \%$ (i.e., from $37.0 \%$ to $39.1 \%$ ) on $A P_{\text {mask }}$ with minor extra inference time (i.e., 10.3 fps v.s. 9.9 fps and 7.6 fps v.s. 7.3 fps). The results show the effectiveness and efficiency of CARAFE++. In Figure 6 we show some examples of instance segmentation results comparing the baseline and CARAFE++.

We further apply CARAFE++ in ResNet-101 backbone with powerful Deformable Convolution layers v2 (DCNv2) [53]. In the ResNet-101 with DCNv2 baseline, the $3 \times 3$ Convolution layers in Res-2, Res-3, Res-4 are replaced with $3 \times 3$ DCNv2. To investigate the effectiveness of CARAFE++, DCNv2 layers with stride of 2 are replaced with CARAFE++ and a following $3 \times 3$ convolution layers without stride. As shown in Table 1, CARAFE++ still achieves substantial gains on such a strong baseline. Specifically, CARAFE++improve $\sim 1 \% A P$ for both Faster R-CNN and Mask R-CNN with similar inference speed.

CARAFE++ for Downsampling. Here we investigate the effectiveness of CARAFE++ for downsampling. As illustrated in Table 2, after integrated into ResNet backbone, CARAFE++ improves the Top- 1 accuracy of image classification on ResNet-50 and ResNet-100 by $1 \%$ and $0.6 \%$ respectively. Therefore, apart from dense predication tasks, CARAFE++ also brings benefits to the classification task.

We further explore different downsampling methods in object detection. To be specific, we adopt different downsampling methods in the ResNet-50 backbone, and then pretrain the backbone on ImageNet-1k classification dataset. The pretrained backbones are used to train Faster R-CNN
TABLE 7: Detection and Instance Segmentation results of Mask R-CNN with CARAFE++ in Backbone, FPN and mask head, respectively. M.H. indicates using CARAFE++ in mask head.

FPN M.H. B.K. Task $\mathrm{AP} \mathrm{AP}_{50} \mathrm{AP}_{75} \mathrm{AP}_{S} \mathrm{AP}_{M} \mathrm{AP}_{L}$

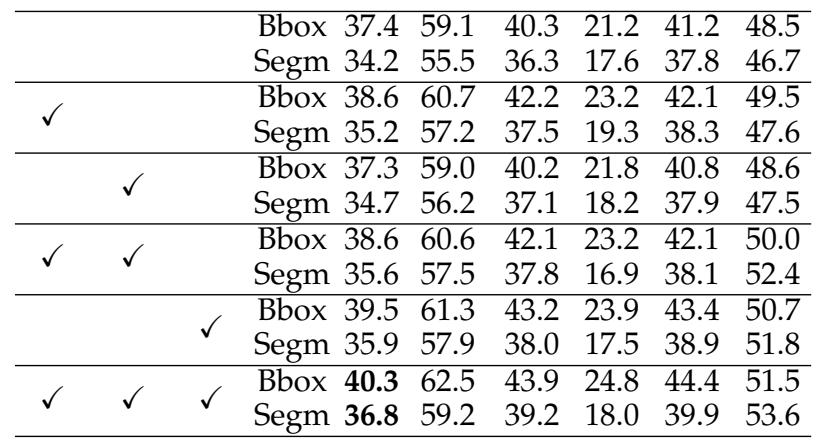

with FPN on COCO dataset. As summarized in Table 3. we extensively compare convolution layer with stride, Max Pooling, Average Pooling, Detail Preserved Pooling [37], Local Importance-based Pooling [9] and CARAFE++. The strided convolution layer is the baseline. For Max Pooling, Average Pooling, Detail Preserved Pooling, we apply these pooling methods in the same manner with CARAFE++ as described in Section 4.1] LIP [9] is adopted following its original paper. We observe that by simply replacing the baseline (i.e., strided convolution layers) with Max/Average/DetailPreserved pooling, the performance can be improved by $\sim 1.3 \%$. The recent proposed LIP achieves a slightly better results than these pooling operators. CARAFE++ significantly improves the baseline by $2.3 \%$ (i.e., from $36.5 \%$ to $38.8 \% \mathrm{AP}$ ) with minor extra cost (i.e., from $12.5 \mathrm{fps}$ to $12.1 \mathrm{fps}$ ).

CARAFE++ for Upsampling. To investigate the effectiveness of different upsampling operators, we perform extensive experiments on Faster R-CNN by using different operators to perform upsampling in FPN. Results are illustrated in Table 4 For 'N.C.' and 'B.C.', which respectively indicate 'Nearest + Conv' and 'Bilinear + Conv', we add an extra $3 \times 3$ convolution layer after the corresponding upsampling. 'Deconv', 'Pixel Shuffle' (indicated as 'P.S.'), 'GUM' are three representative learning-based upsampling methods. We also compare 'Spatial Attention' here, indicated as 'S.A.'. CARAFE++ achieves the best $A P_{b o x}$ among all these upsampling operators with minor extra computation cost, which illustrates it is both effective and efficient. The results of 'Nearest + Conv' and 'Bilinear + Conv' show that simply adding more convolution layers does not lead to a significant gain. However, it slows down the inference speed from $12.5 \mathrm{fps}$ to $11.8 \mathrm{fps}$. 'Deconv', 'Pixel Shuffle', 'GUM' and 'Spatial Attention' obtain inferior performance to CARAFE++, indicating that the design of effective upsampling operators is critical.

In Table 5, we report the object detection performance of adopting CARAFE++ in Backbone and FPN, respectively. Adopting CARAFE++ both in Backbone and FPN further improves the object detection performance to $39.6 \%$.

CARAFE++ for Mask Predication. Besides FPN, which is a pyramid feature fusion structure, we also explore different upsampling operators in the mask head. In typical Mask $\mathrm{R}-\mathrm{CNN}$, a deconvolution layer is adopted to upsample the RoI features by $2 x$. For a fair comparison, we do not make 


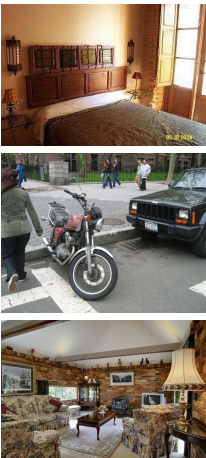

Input Image

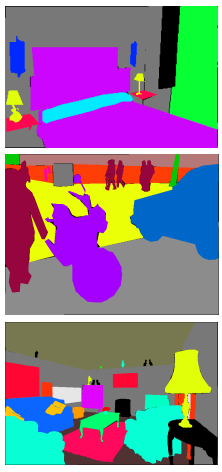

Ground Truth

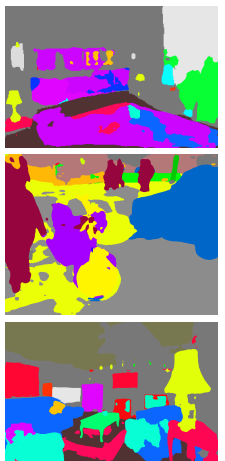

UperNet

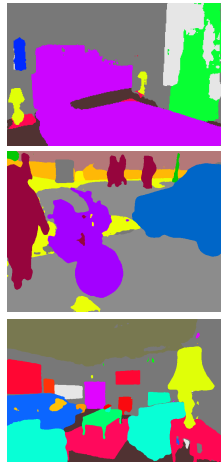

UperNet w/ CARAFE++

Fig. 7: Comparison of semantic segmentation results between UperNet baseline and UperNet w/ CARAFE++ on ADE20k.

TABLE 8: Semantic Segmentation results on ADE20k val. Single scale testing is used in our experiments. P.A. indicates Pixel Accuracy.

\begin{tabular}{cccc}
\hline Method & Backbone & mIoU & \multirow{2}{*}{ P.A. } \\
\hline PSPNet & ResNet-50 & 41.68 & 80.04 \\
PSANet & ResNet-50 & 41.92 & 80.17 \\
UperNet $2^{2}$ & ResNet-50 & 40.44 & 79.80 \\
UperNet w/ CARAFE++ & ResNet-50 & $\mathbf{4 3 . 0 5}$ & $\mathbf{8 0 . 8 1}$ \\
\hline PSPNet & ResNet-101 & 41.96 & 80.64 \\
PSANet & ResNet-101 & 42.75 & 80.71 \\
UperNet & ResNet-101 & 42.00 & 80.79 \\
UperNet w/ CARAFE++ & ResNet-101 & $\mathbf{4 3 . 9 4}$ & $\mathbf{8 1 . 2 6}$ \\
\hline
\end{tabular}

any changes to FPN, and only replace the deconvolution layer with various operators. Since we only modify the mask prediction branch, performance is reported in terms of $A P_{\text {mask }}$, as shown in Table 6. CARAFE++ achieves the best performance in instance segmentation among these methods.

In Table 7, we report the object detection and instance segmentation performance of adopting CARAFE++ in Backbone, FPN and mask head on Mask R-CNN, respectively. Consistent improvements are achieved in these experiments.

Semantic Segmentation. We replace the downsamplers and upsamplers in UperNet with CARAFE++ and evaluate the results on ADE20k benchmark. As shown in Table 8, adopting single scale testing, CARAFE++ improves the mIoU by a large margin from $40.44 \%$ to $43.05 \%$ on ResNet-50 Backbone and from $42.0 \%$ to $43.94 \%$ with ResNet-101 backbone. Note that UperNet with CARAFE++ also achieves better performance than other strong baselines such as PSPNet [47| and PSANet [48].

We perform a step-by-step study to inspect the effectiveness of modifying different components in UperNet, as described in Section 4.2 Results in Table 9 show that CARAFE++ is helpful for all the four components and the combination of them results in further gains. Visualization comparisons of UperNet with and without CARAFE++ are shown in Figure 7

2. We report the performance in model zoo of the official implementation:

https://github.com/CSAILVision/semantic-segmentation-pytorch

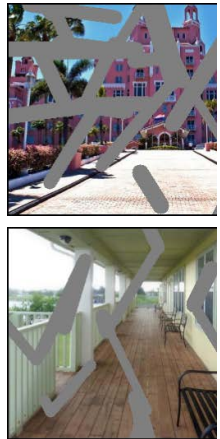

Masked Input

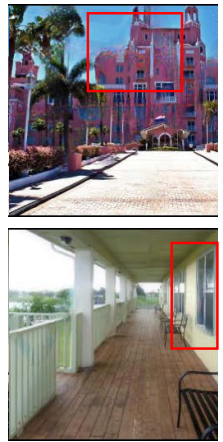

Global\&Local
Global\&Local w/ CARAFE++

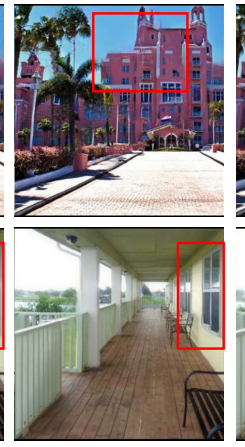

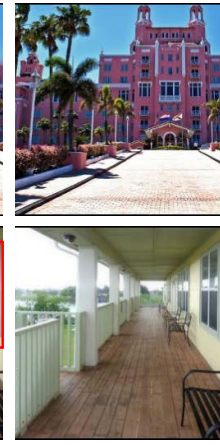

Original Image
Fig. 8: Comparison of image inpainting results between Gobal\&Local baseline and Gobal\&Local w/ CARAFE++ on ADE20k.

TABLE 9: Effects of adopting CARAFE++ in each component of UperNet.

\begin{tabular}{cccccc}
\hline PPM & FPN & FUSE & Backbone & mIoU & P.A. \\
\hline$\checkmark$ & & & & 40.85 & 79.97 \\
& $\checkmark$ & & & 40.79 & 80.01 \\
& & $\checkmark$ & & 41.06 & 80.23 \\
$\checkmark$ & $\checkmark$ & & & 41.55 & 80.30 \\
$\checkmark$ & & $\checkmark$ & & 42.01 & 80.11 \\
& $\checkmark$ & $\checkmark$ & & 41.93 & 80.34 \\
$\checkmark$ & $\checkmark$ & $\checkmark$ & & 42.23 & 80.34 \\
& & & $\checkmark$ & 41.93 & 80.35 \\
$\checkmark$ & $\checkmark$ & $\checkmark$ & $\checkmark$ & $\mathbf{4 3 . 0 5}$ & $\mathbf{8 0 . 8 1}$ \\
\hline
\end{tabular}

Image Inpainting. We show that CARAFE++ is also effective in low-level tasks such as image inpainting. By replacing the upsampling operators with CARAFE++ in two strong baselines Global\&Local [15] and Partial Conv [26], we observe substantial improvements for both methods. As shown in Table 10 our method improves two baselines by $1.35 \mathrm{~dB}$ and $0.27 \mathrm{~dB}$ on the PSNR metric. Visualization results of CARAFE++ are shown in Figure 8

\subsection{Ablation Study \& Further Analysis}

Model Design \& Hyper-parameters. We investigate the influence of hyper-parameters in the model design, i.e., the compressed channels $C_{m}$, encoder kernel size $k_{\text {encoder }}$ and reassembly kernel size $k_{\text {reassembly }}$. We also test different normalization methods in the kernel normalizer. Faster $\mathrm{R}-\mathrm{CNN}$ with a ResNet-50 backbone and $1 \mathrm{x}$ schedule is adopted for ablation study if not further specified.

We explore different values of $C_{m}$ in the channel compressor. The experiments are conducted on object detection for upsampling and image classification for downsampling. For the upsampling version of CARAFE++, the influences of $C_{m}$ is evaluated on Faster R-CNN with ResNet-50 backbone. Experimental results in Table 11 show that compress $C_{m}$ down to 64 leads to no performance decline, while being more efficient. A further smaller $C_{m}$ will result in a slight drop of the performance. In addition, we also try removing the channel compressor module, which means the content encoder directly uses input features to predict reassembly kernels. With no channel compressor, it can achieve the same performance, suggesting the capability of the channel compressor in speeding up the kernel prediction without 

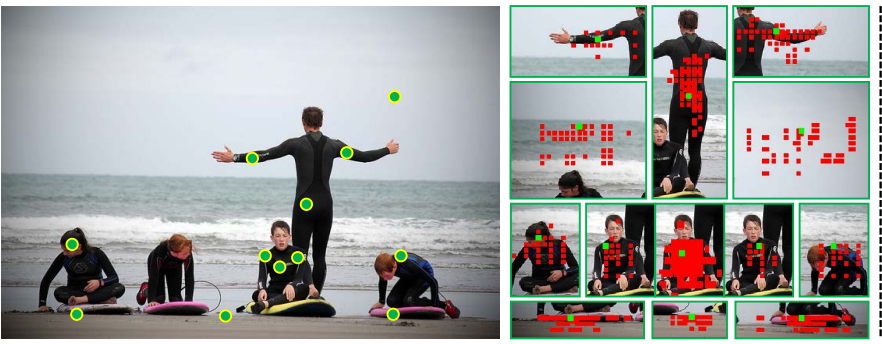

(a) CARAFE++ Upsample
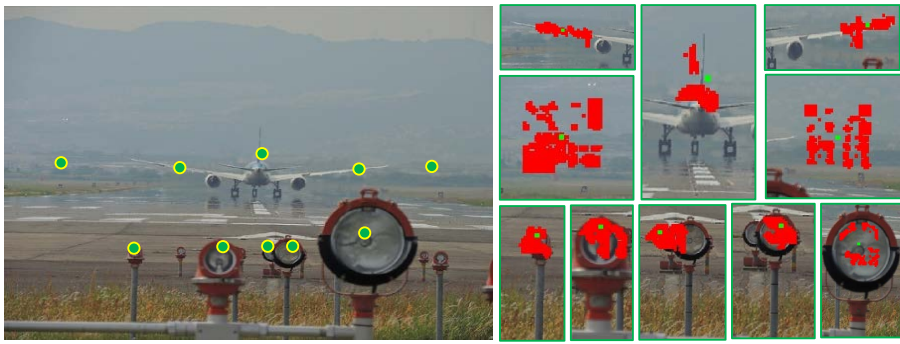

(b) CARAFE++ Downsample

Example Locations Reassembly Center Reassembled Units

Fig. 9: CARAFE++ performs content-aware reassembly when rescaling a feature map. Red units are reassembled into the green center unit by CARAFE++.

TABLE 10: Image inpainting results on Places val.

\begin{tabular}{lll}
\hline Method & L1(\%) & PSNR(dB) \\
\hline Global\&Local & 6.69 & 19.58 \\
Global\&Local w / CARAFE++ & 5.82 & 20.93 \\
\hline Partial Conv & 5.96 & 20.78 \\
Partial Conv w / CARAFE++ & $\mathbf{5 . 6 0}$ & $\mathbf{2 1 . 0 5}$ \\
\hline
\end{tabular}

TABLE 11: Ablation study of various compressed channels $C_{m}$ for upsampling. The experiments are performed with Faster R$\mathrm{CNN}$ w/ CARAFE++in FPN structure on COCO dataset. N/A means channel compressor is removed.

\begin{tabular}{lllllll}
\hline$C_{m}$ & $\mathrm{AP}$ & $\mathrm{AP}_{50}$ & $\mathrm{AP}_{75}$ & $\mathrm{AP}_{S}$ & $\mathrm{AP}_{M}$ & $\mathrm{AP}_{L}$ \\
\hline 16 & 37.6 & 60.1 & 40.6 & 22.7 & 41.6 & 48.4 \\
32 & 37.7 & 60.3 & 40.7 & 22.8 & 41.2 & 49.0 \\
64 & $\mathbf{3 7 . 8}$ & 60.1 & 40.8 & 23.2 & 41.2 & 48.2 \\
128 & $\mathbf{3 7 . 8}$ & 60.1 & 40.8 & 22.4 & 41.7 & 48.7 \\
256 & $\mathbf{3 7 . 8}$ & 60.4 & 40.8 & 22.7 & 41.3 & 48.8 \\
$\mathrm{~N} / \mathrm{A}$ & $\mathbf{3 7 . 8}$ & 60.3 & 40.8 & 22.9 & 41.5 & 48.7 \\
\hline
\end{tabular}

harming the performance. Based on the above results, we set $C_{m}$ to 64 by default as a trade-off between performance and efficiency.

For the downsampling version of CARAFE++, we adopt different settings of $C_{m}$ in a ResNet-50 for image classification. As shown in Table 12, with different $C_{m}$, the classification accuracy is relatively stable. We choose $C_{m}=16$ that achieves the best performance and involves less computational cost.

We then investigate the influence of $k_{\text {encoder }}$ and $k_{\text {reassembly }}$. Intuitively, increasing $k_{\text {reassembly }}$ also requires a larger $k_{\text {encoder }}$ since the content encoder needs a large receptive field to predict a large reassembly kernel. As illustrated in Table 13 . increasing $k_{\text {encoder }}$ and $k_{\text {reassembly }}$ at the same time can boost the performance, while just enlarging one of them will not. We summarize an empirical formula that $k_{\text {encoder }}=k_{\text {reassembly }}-2$, which is a good choice in all the settings. Though adopting a larger kernel size is shown helpful, we set $k_{\text {reassembly }}=5$ and $k_{\text {encoder }}=3$ by default as a trade-off between performance and efficiency.

Other than the softmax function, we also test other alternatives in the kernel normalizer, such as sigmoid or sigmoid with normalization. As shown in Table 14. 'Softmax' and 'Sigmoid Normalized' have the same performance and better than 'Sigmoid', which shows that it is crucial to normalize the reassembly kernel to be summed to 1 .

How CARAFE++ Works. We conduct a further qualitative study to figure out how CARAFE++ works for upsampling
TABLE 12: Ablation study of various compressed channels $C_{m}$ for downsampling. The experiments are performed with ResNet-50 on ImageNet-1k classification dataset.

\begin{tabular}{ccc}
\hline$C_{m}$ & Top-1 & Top-5 \\
\hline 4 & 77.31 & 93.59 \\
8 & 77.43 & 93.63 \\
16 & 77.46 & 93.63 \\
32 & 77.40 & 93.60 \\
64 & 77.46 & 93.67 \\
\hline
\end{tabular}

TABLE 13: Detection results with various encoder kernel size $k_{\text {encoder }}$ and reassembly kernel size $k_{\text {reassembly }}$.

\begin{tabular}{llllllll}
\hline \multirow{2}{*}{$k_{\text {encoder }}$} & $k_{\text {reassembly }}$ & $\mathrm{AP}$ & $\mathrm{AP}_{50}$ & $\mathrm{AP}_{75}$ & $\mathrm{AP}_{S}$ & $\mathrm{AP}_{M}$ & $\mathrm{AP}_{L}$ \\
\hline 1 & 3 & 37.3 & 59.6 & 40.5 & 22.0 & 40.7 & 48.1 \\
1 & 5 & 37.3 & 59.9 & 40.0 & 22.3 & 41.1 & 47.3 \\
3 & 3 & 37.3 & 59.7 & 40.4 & 22.1 & 40.8 & 48.3 \\
3 & 5 & 37.8 & 60.1 & 40.8 & 23.2 & 41.2 & 48.2 \\
3 & 7 & 37.7 & 60.0 & 40.9 & 23.0 & 41.5 & 48.4 \\
5 & 5 & 37.8 & 60.2 & 40.7 & 22.5 & 41.4 & 48.6 \\
5 & 7 & 38.1 & 60.4 & 41.3 & 23.0 & 41.6 & 48.8 \\
7 & 7 & 38.0 & 60.2 & 41.1 & 23.0 & 41.8 & 48.8 \\
\hline
\end{tabular}

and downsampling. With trained Mask R-CNN models that adopts CARAFE++ as the upsampling and downsampling operator, respectively, we visualize the reassembling process in Figure 9. Specifically, we sample some pixels in the feature map that the upsampling/downsampling process attained, and see which neighbors it is reassembled from. The green circle denotes example locations and red dots indicate highly weighted sources during the reassembly. For upsampling (see Figure 9(a)), in the FPN structure, the lowresolution feature map will be consecutively upsampled for several times to a higher resolution, so a pixel in the upsampled feature map reassembles information from a more larger region. For downsampling (see Figure 9 (b)), a input high-resolution feature map is downsampled for several times by CARAFE++. In the process, pixels in a large region of the high-resolution feature map is reassembled to attain a pixel on the low-resolution feature map. As a result, the receptive field of a downsampled feature map increases. From the figure, we can clearly learn that CARAFE++ is content-aware. It tends to reassemble points with similar semantic information. A location at the foreground instance, e.g., human body and wing of an airplane, prefers other points from the same instance, rather than other objects or nearby background. For locations in the background regions which has weaker semantics, the reassembly is more 
TABLE 14: Ablation study of different normalization methods in kernel normalizer.

\begin{tabular}{lllllll}
\hline Method & $\mathrm{AP}$ & $\mathrm{AP}_{50}$ & $\mathrm{AP}_{75}$ & $\mathrm{AP}_{S}$ & $\mathrm{AP}_{M}$ & $\mathrm{AP}_{L}$ \\
\hline Sigmoid & 37.4 & 59.8 & 40.2 & 23.1 & 40.9 & 47.4 \\
Sigmoid Normalize & $\mathbf{3 7 . 8}$ & 60.1 & 40.7 & 22.6 & 41.6 & 48.0 \\
Softmax & $\mathbf{3 7 . 8}$ & 60.1 & 40.8 & 23.2 & 41.2 & 48.2 \\
\hline
\end{tabular}

uniform or just biased on points with similar low-level texture features.

\section{Conclusion}

We have presented Unified Content-Aware ReAssembly of FEatures (CARAFE++), a universal, lightweight and highly effective feature reassembly operator. It consistently boosts the performances on standard benchmarks in object detection, instance/semantic segmentation and inpainting by $2.5 \% A P_{\text {box }}, 2.1 \% A P_{\text {mask }}, 1.94 \%$ mIoU, $1.35 \mathrm{~dB}$, respectively. More importantly, CARAFE++ introduces little computational overhead and can be readily integrated into modern network architectures. It shows great potential to serve as a strong building block for future research. Moreover, the current version of CARAFE++ supports feature upsampling/downsampling by an integer factor. In our future work, CARAFE++ will support feature reassembly with an arbitrary scale factor. Then it could be more widely integrated for various network architectures.

Acknowledgements. This work is partially supported by the Collaborative Research Grant from SenseTime Group (CUHK Agreement No. TS1610626 \& No. TS1712093), the General Research Fund (GRF) of Hong Kong (No. 14236516 \& No. 14203518), Singapore MOE AcRF Tier 1 (M4012082.020), NTU SUG, and NTU NAP.

\section{RefERENCES}

[1] Kai Chen, Jiangmiao Pang, Jiaqi Wang, Yu Xiong, Xiaoxiao Li, Shuyang Sun, Wansen Feng, Ziwei Liu, Jianping Shi, Wanli Ouyang, Chen Change Loy, and Dahua Lin. Hybrid task cascade for instance segmentation. In IEEE Conference on Computer Vision and Pattern Recognition, 2019.

[2] Kai Chen, Jiaqi Wang, Jiangmiao Pang, Yuhang Cao, Yu Xiong, Xiaoxiao Li, Shuyang Sun, Wansen Feng, Ziwei Liu, Jiarui Xu, Zheng Zhang, Dazhi Cheng, Chenchen Zhu, Tianheng Cheng, Qijie Zhao, Buyu Li, Xin Lu, Rui Zhu, Yue Wu, Jifeng Dai, Jingdong Wang, Jianping Shi, Wanli Ouyang, Chen Change Loy, and Dahua Lin. MMDetection: Open mmlab detection toolbox and benchmark. arXiv preprint arXiv:1906.07155, 2019.

[3] Long Chen, Hanwang Zhang, Jun Xiao, Liqiang Nie, Jian Shao, Wei Liu, and Tat-Seng Chua. SCA-CNN: Spatial and channel-wise attention in convolutional networks for image captioning. In IEEE Conference on Computer Vision and Pattern Recognition, 2017.

[4] Liang-Chieh Chen, George Papandreou, Iasonas Kokkinos, Kevin Murphy, and Alan L Yuille. Deeplab: Semantic image segmentation with deep convolutional nets, atrous convolution, and fully connected crfs. IEEE Transactions on Pattern Analysis and Machine Intelligence, 40(4):834-848, 2018.

[5] Liang-Chieh Chen, Yukun Zhu, George Papandreou, Florian Schroff, and Hartwig Adam. Encoder-decoder with atrous separable convolution for semantic image segmentation. In European Conference on Computer Vision, 2018.

[6] Jifeng Dai, Haozhi Qi, Yuwen Xiong, Yi Li, Guodong Zhang, Han $\mathrm{Hu}$, and Yichen Wei. Deformable convolutional networks. In IEEE International Conference on Computer Vision, 2017.

[7] Jia Deng, Wei Dong, Richard Socher, Li-Jia Li, Kai Li, and Li FeiFei. Imagenet: A large-scale hierarchical image database. In IEEE Conference on Computer Vision and Pattern Recognition, 2009.
[8] Chao Dong, Chen Change Loy, Kaiming He, and Xiaoou Tang. Image super-resolution using deep convolutional networks. IEEE Transactions on Pattern Analysis and Machine Intelligence, 38(2):295307, 2016.

[9] Ziteng Gao, Limin Wang, and Gangshan Wu. Lip: Local importance-based pooling. In IEEE International Conference on Computer Vision, October 2019.

[10] Ross Girshick, Ilija Radosavovic, Georgia Gkioxari, Piotr Dollár, and Kaiming He. Detectron. https://github.com/ facebookresearch/detectron 2018.

[11] Priya Goyal, Piotr Dollár, Ross Girshick, Pieter Noordhuis, Lukasz Wesolowski, Aapo Kyrola, Andrew Tulloch, Yangqing Jia, and Kaiming He. Accurate, large minibatch sgd: Training imagenet in 1 hour. arXiv preprint arXiv:1706.02677, 2017.

[12] Kaiming He, Georgia Gkioxari, Piotr Dollár, and Ross Girshick. Mask R-CNN. In IEEE International Conference on Computer Vision, 2017.

[13] Kaiming He, Xiangyu Zhang, Shaoqing Ren, and Jian Sun. Deep residual learning for image recognition. In IEEE Conference on Computer Vision and Pattern Recognition, 2016.

[14] Xuecai Hu, Haoyuan Mu, Xiangyu Zhang, Zilei Wang, Tieniu Tan, and Jian Sun. Meta-SR: A magnification-arbitrary network for super-resolution. In IEEE Conference on Computer Vision and Pattern Recognition, 2019.

[15] Satoshi Iizuka, Edgar Simo-Serra, and Hiroshi Ishikawa. Globally and locally consistent image completion. ACM Transactions on Graphics, 36(4):107, 2017.

[16] Max Jaderberg, Karen Simonyan, Andrew Zisserman, et al. Spatial transformer networks. In Advances in Neural Information Processing Systems, 2015.

[17] Xu Jia, Bert De Brabandere, Tinne Tuytelaars, and Luc V Gool. Dynamic filter networks. In Advances in Neural Information Processing Systems, 2016.

[18] Younghyun Jo, Seoung Wug Oh, Jaeyeon Kang, and Seon Joo Kim. Deep video super-resolution network using dynamic upsampling filters without explicit motion compensation. In IEEE Conference on Computer Vision and Pattern Recognition, 2018.

[19] Tao Kong, Fuchun Sun, Chuanqi Tan, Huaping Liu, and Wenbing Huang. Deep feature pyramid reconfiguration for object detection. In European Conference on Computer Vision, 2018.

[20] Chuan Li and Michael Wand. Precomputed real-time texture synthesis with markovian generative adversarial networks. In European Conference on Computer Vision, 2016.

[21] Xiaoxiao Li, Ziwei Liu, Ping Luo, Chen Change Loy, and Xiaoou Tang. Not all pixels are equal: difficulty-aware semantic segmentation via deep layer cascade. In IEEE Conference on Computer Vision and Pattern Recognition, 2017.

[22] Bee Lim, Sanghyun Son, Heewon Kim, Seungjun Nah, and Kyoung $\mathrm{Mu}$ Lee. Enhanced deep residual networks for single image super-resolution. In IEEE Conference on Computer Vision and Pattern Recognition Workshop, 2017.

[23] Tsung-Yi Lin, Piotr Dollar, Ross Girshick, Kaiming He, Bharath Hariharan, and Serge Belongie. Feature pyramid networks for object detection. In IEEE Conference on Computer Vision and Pattern Recognition, July 2017.

[24] Tsung-Yi Lin, Priya Goyal, Ross Girshick, Kaiming He, and Piotr Dollár. Focal loss for dense object detection. In IEEE International Conference on Computer Vision, 2017.

[25] Tsung-Yi Lin, Michael Maire, Serge Belongie, James Hays, Pietro Perona, Deva Ramanan, Piotr Dollár, and C Lawrence Zitnick. Microsoft COCO: Common objects in context. In European Conference on Computer Vision, 2014.

[26] Guilin Liu, Fitsum A Reda, Kevin J Shih, Ting-Chun Wang, Andrew Tao, and Bryan Catanzaro. Image inpainting for irregular holes using partial convolutions. In European Conference on Computer Vision, 2018.

[27] Shu Liu, Lu Qi, Haifang Qin, Jianping Shi, and Jiaya Jia. Path aggregation network for instance segmentation. In IEEE Conference on Computer Vision and Pattern Recognition, 2018.

[28] Wei Liu, Dragomir Anguelov, Dumitru Erhan, Christian Szegedy, Scott Reed, Cheng-Yang Fu, and Alexander C Berg. Ssd: Single shot multibox detector. In European Conference on Computer Vision, 2016.

[29] Ziwei Liu, Xiaoxiao Li, Ping Luo, Chen-Change Loy, and Xiaoou Tang. Semantic image segmentation via deep parsing network. In IEEE International Conference on Computer Vision, 2015.

[30] Davide Mazzini. Guided upsampling network for real-time semantic segmentation. arXiv preprint arXiv:1807.07466, 2018.

[31] Ben Mildenhall, Jonathan T Barron, Jiawen Chen, Dillon Sharlet, Ren $\mathrm{Ng}$, and Robert Carroll. Burst denoising with kernel predic- 
tion networks. In IEEE Conference on Computer Vision and Pattern Recognition, 2018.

[32] Alejandro Newell, Kaiyu Yang, and Jia Deng. Stacked hourglass networks for human pose estimation. In European Conference on Computer Vision, 2016.

[33] Hyeonwoo Noh, Seunghoon Hong, and Bohyung Han. Learning deconvolution network for semantic segmentation. IEEE International Conference on Computer Vision, Dec 2015.

[34] Deepak Pathak, Philipp Krahenbuhl, Jeff Donahue, Trevor Darrell, and Alexei A Efros. Context encoders: Feature learning by inpainting. In IEEE Conference on Computer Vision and Pattern Recognition, 2016.

[35] Shaoqing Ren, Kaiming He, Ross Girshick, and Jian Sun. Faster R-CNN: Towards real-time object detection with region proposal networks. In Advances in Neural Information Processing Systems, 2015.

[36] Olaf Ronneberger, Philipp Fischer, and Thomas Brox. U-net: Convolutional networks for biomedical image segmentation. In International Conference on Medical image computing and computerassisted intervention, 2015.

[37] Faraz Saeedan, Nicolas Weber, Michael Goesele, and Stefan Roth. Detail-preserving pooling in deep networks. In IEEE Conference on Computer Vision and Pattern Recognition, June 2018.

[38] Wenzhe Shi, Jose Caballero, Ferenc Huszár, Johannes Totz, Andrew P Aitken, Rob Bishop, Daniel Rueckert, and Zehan Wang. Real-time single image and video super-resolution using an efficient sub-pixel convolutional neural network. In IEEE Conference on Computer Vision and Pattern Recognition, 2016.

[39] Dmitry Ulyanov, Andrea Vedaldi, and Victor Lempitsky. Deep image prior. In IEEE Conference on Computer Vision and Pattern Recognition, 2018

[40] Jiaqi Wang, Kai Chen, Rui Xu, Ziwei Liu, Chen Change Loy, and Dahua Lin. Carafe: Content-aware reassembly of features. In The IEEE International Conference on Computer Vision (ICCV), October 2019.

[41] Jiaqi Wang, Kai Chen, Shuo Yang, Chen Change Loy, and Dahua Lin. Region proposal by guided anchoring. In IEEE Conference on Computer Vision and Pattern Recognition, 2019.

[42] Jiaqi Wang, Wenwei Zhang, Yuhang Cao, Kai Chen, Jiangmiao Pang, Tao Gong, Jianping Shi, Chen Change Loy, and Dahua Lin. Side-aware boundary localization for more precise object detection. In $E C C V, 2020$.

[43] Tete Xiao, Yingcheng Liu, Bolei Zhou, Yuning Jiang, and Jian Sun. Unified perceptual parsing for scene understanding. In European Conference on Computer Vision, 2018.

[44] Rui Xu, Xiaoxiao Li, Bolei Zhou, and Chen Change Loy. Deep flow-guided video inpainting. In IEEE Conference on Computer Vision and Pattern Recognition, June 2019.

[45] Jiahui Yu, Zhe Lin, Jimei Yang, Xiaohui Shen, Xin Lu, and Thomas S Huang. Free-form image inpainting with gated convolution. arXiv preprint arXiv:1806.03589, 2018.

[46] Jiahui Yu, Zhe Lin, Jimei Yang, Xiaohui Shen, Xin Lu, and Thomas $S$ Huang. Generative image inpainting with contextual attention. In IEEE Conference on Computer Vision and Pattern Recognition, 2018.

[47] Hengshuang Zhao, Jianping Shi, Xiaojuan Qi, Xiaogang Wang, and Jiaya Jia. Pyramid scene parsing network. In IEEE Conference on Computer Vision and Pattern Recognition, 2017.

[48] Hengshuang Zhao, Yi Zhang, Shu Liu, Jianping Shi, Chen Change Loy, Dahua Lin, and Jiaya Jia. PSANet: Point-wise spatial attention network for scene parsing. In European Conference on Computer Vision, 2018.

[49] Qijie Zhao, Tao Sheng, Yongtao Wang, Zhi Tang, Ying Chen, Ling Cai, and Haibin Ling. M2Det: A single-shot object detector based on multi-level feature pyramid network. In AAAI Conference on Artificial Intelligence, 2019.

[50] Bolei Zhou, Agata Lapedriza, Aditya Khosla, Aude Oliva, and Antonio Torralba. Places: A 10 million image database for scene recognition. IEEE Transactions on Pattern Analysis and Machine Intelligence, 2017.

[51] Bolei Zhou, Hang Zhao, Xavier Puig, Sanja Fidler, Adela Barriuso, and Antonio Torralba. Scene parsing through ADE20K dataset. In IEEE Conference on Computer Vision and Pattern Recognition, 2017.

[52] Bolei Zhou, Hang Zhao, Xavier Puig, Tete Xiao, Sanja Fidler, Adela Barriuso, and Antonio Torralba. Semantic understanding of scenes through the ade20k dataset. International Journal of Computer Vision, 2018.

[53] Xizhou Zhu, Han Hu, Stephen Lin, and Jifeng Dai. Deformable convnets v2: More deformable, better results. In IEEE Conference on Computer Vision and Pattern Recognition, June 2019.
Jiaqi Wang is a fourth-year Ph.D. candidate in Multimedia Laboratory (MMLab) at the Chinese University of Hong Kong. He is supervised by Prof. Dahua Lin and works closely with Prof. Chen Change Loy. He received the B.Eng. degree from Sun Yat-Sen University in 2017 and began to pursue his Ph.D. degree funded by Hong Kong PhD Fellowship Scheme (HKPFS) in the same year. His research interests focus on object detection and instance segmentation.

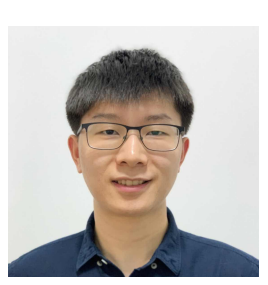

Kai Chen is a vice director at SenseTime, leading the algorithm platform team of EIG Research. He received the $\mathrm{PhD}$ degree in The Chinese University of Hong Kong in 2019, under the supervision of Prof. Dahua Lin and Chen Change Loy at MMLab. Before that, he received the B.Eng. degree from Tsinghua University in 2015. His research interests include computer vision and deep learning, particularly focusing on object detection, segmentation and video understanding.

Rui $\mathrm{Xu}$ received the BEng degree in electronic engineering from Tsinghua University, in 2018. $\mathrm{He}$ is working toward the $\mathrm{PhD}$ degree in Multimedia Laboratory (MMLab) at the Chinese University of Hong Kong (CUHK). His research interests include computer vision and deep learning especially for several topics in low-level vision like inpainting and image generation.

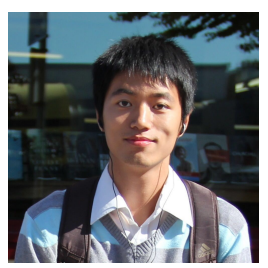

Ziwei Liu Ziwei Liu is currently a Nanyang Assistant Professor at Nanyang Technological University (NTU). Previously, he was a senior research fellow at the Chinese University of Hong Kong. Before that, Ziwei was a postdoctoral researcher at University of California, Berkeley, working with Prof. Stella Yu. Ziwei received his PhD from the Chinese University of Hong Kong in 2017, under the supervision of Prof. Xiaoou Tang and Prof. Xiaogang Wang. He has published over 50 papers (with more than 7,000 citations) on toptier conferences and journals in relevant fields, including CVPR, ICCV, ECCV, AAAI, IROS, SIGGRAPH, T-PAMI, and TOG. He is the recipient of Microsoft Young Fellowship, Hong Kong PhD Fellowship, ICCV Young Researcher Award, and HKSTP best paper award.

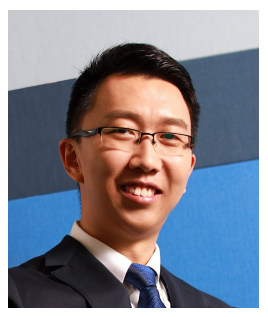

Chen Change Loy (Senior Member, IEEE) received the $\mathrm{PhD}$ degree in computer science from the Queen Mary University of London, in 2010. $\mathrm{He}$ is an associate professor with the School of Computer Science and Engineering, Nanyang Technological University. Prior to joining NTU, he served as a research assistant professor with the Department of Information Engineering, The Chinese University of Hong Kong, from 2013 to 2018. His research interests include computer vision and deep learning. He serves as an associate editor of the IEEE Transactions on Pattern Analysis and Machine Intelligence and the International Journal of Computer Vision. He also serves/served as an Area Chair of ICCV 2021, CVPR 2021, CVPR 2019, ECCV 2018, AAAI 2021 and BMVC 2018-2020. 


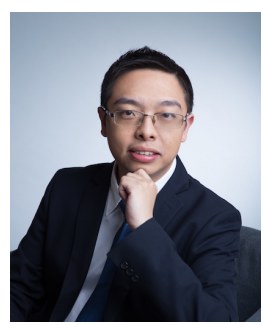

Dahua Lin Dahua Lin is an Associate Professor at the department of Information Engineering, the Chinese University of Hong Kong, and the Director of CUHK-SenseTime Joint Laboratory. $\mathrm{He}$ received the B.Eng. degree from the University of Science and Technology of China (USTC) in 2004, the M. Phil. degree from the Chinese University of Hong Kong (CUHK) in 2006, and the Ph.D. degree from Massachusetts Institute of Technology (MIT) in 2012. Prior to joining CUHK, he served as a Research Assistant Professor at Toyota Technological Institute at Chicago, from 2012 to 2014. His research interest covers computer vision and machine learning. He serves on the editorial board of the International Journel of Computer Vision (IJCV). He also serves as an area chair for multiple conferences, including ECCV 2018, ACM Multimedia 2018, BMVC 2018, CVPR 2019 , BMVC 2019, AAAI 2020, and CVPR 2021. 